Proceeding Series of the Brazilian Society of Computational and Applied Mathematics

\title{
Inclusão da Não Linearidade de Atrito na Modelagem Matemática da Dinâmica de um Robô Gantry com Transmissão do Tipo Fuso de Esferas
}

\author{
Angelo Fernando Fiori ${ }^{1}$ \\ Ismael Barbieri Garlet ${ }^{2}$ \\ Odmartan Ribas Maciel ${ }^{3}$ \\ Andrei Fiegenbaum ${ }^{4}$ \\ Leonardo Bortolon Maraschin \\ Antonio Carlos Valdiero ${ }^{6}$ \\ Luiz Antonio Rasia ${ }^{7}$ \\ Departamento de Ciências Exatas e Engenharias, UNIJUÍ, Panambi, RS
}

\begin{abstract}
Resumo. Apresenta-se a identificação experimental da não linearidade do atrito em uma transmissão mecânica do tipo fuso de esferas no protótipo de uma junta do robô Gantry acionado por motorredutores de corrente alternada e inversores de frequência e a posterior inclusão do mapa estático na validação de seu modelo dinâmico.
\end{abstract}

Palavras-chave. Robôs de Pórtico, Acionamento Elétrico, Validação Experimental, Modelo Dinâmico, Identificação Experimental.

\section{Introdução}

Os sistemas robóticos, em especial os de pórtico (Gantry), vêm se tornando cada vez mais complexos e mais caros [5]. Os robôs do tipo Gantry são os mais robustos e

\footnotetext{
1 an@unochapeco.edu.br

2 ismael.garlet@hotmail.com

3 odeijui@ hotmail.com

4 andrei.fig@ hotmail.com

5 leonardo.maraschin@unijui.edu.br

6 valdiero@unijui.edu.br

7 rasia@unijui.edu.br
} 
possuem uma cinemática mais simples por se utilizar juntas prismáticas, com eixos perpendiculares, sendo muito aplicados pela facilidade de programação [4] especialmente nas funções de guincho robô, usinagem $\mathrm{CNC}$ e cortes a laser.

No entanto, o controle preciso de robôs Gantry para aplicações depende da identificação e inclusão de não linearidades relevantes como o atrito às quais são pouco estudadas enquanto pesquisa teórica-experimental, sendo um problema amplo e em aberto. Diversas pesquisas ([2], [3], [8]), tem se esforçado em desenvolver estratégias mais precisas de controle e estudar dinâmicas e aspectos de projeto, sendo o diferencial deste trabalho a reunião de diferentes olhares sob o problema da modelagem dinâmica de sistemas mecânicos em robôs: modelagem matemática, testes de bancada, validação experimental, estudo de características constitutivas e inclusão da não linearidade do atrito no modelo dinâmico do robô Gantry.

Assim, tem-se por objetivo neste trabalho incluir a não linearidade de atrito na modelagem dinâmica do protótipo de uma junta de robô do tipo Gantry acionado por inversor de frequência e motorredutor de corrente alternada. Para isso foi construído um protótipo, desenvolvida uma metodologia para a identificação experimental das características do atrito (as quais estão apresentadas no mapa estático de atrito) e posteriormente feita à inclusão destas características no modelo dinâmico do robô Gantry o qual é validado experimentalmente.

\section{Propósito}

As características não lineares do atrito estão presentes em todos os mecanismos mecânicos que incorporem movimentos. Estudá-lo e compreendê-lo é importante para perceber seus efeitos em tais mecanismos, de modo a compensar e por consequência diminuir seus efeitos. O atrito gera erros/ciclos limites no seguimento de trajetórias causando efeitos de aderência-deslizamento (que alterna movimentos e repousos), oscilações (o movimento varia em torno de uma dada posição constante), perda de movimento (ocorre quando o sistema é detido no repouso em um intervalo de tempo onde a velocidade é nula), falha de quadratura (desvios do seguimento de um movimento de múltiplos eixos), dentre outras dificuldades para o controle e a estabilidade destacadas pelos autores e que são geradas pelo atrito [1].

O atrito exibe diversas características clássicas compostas pelo atrito estático, atrito de Coulomb, atrito viscoso e o atrito de arraste, baseados em mapas estáticos e características dinâmicas mais complexas como o atrito de Stribeck, atrito estático crescente, memória do atrito e o deslocamento de predeslizamento ([6] e [9]). As características do atrito dependem geralmente da velocidade, temperatura, sentido do movimento, lubrificação e desgaste das superfícies, da posição e da história do movimento, de modo que a escolha do modelo de atrito apropriado depende das características apresentadas [6].

A combinação das características de atrito (atrito estático, de Coulomb, viscoso, de arraste e a curva de Stribeck), resultam em uma função não linear semelhante a representada na Figura 1 onde são relacionadas a força de atrito $\left(F_{a t r}\right)$ e a velocidade em regime permanente $(\dot{y}(t))$. 


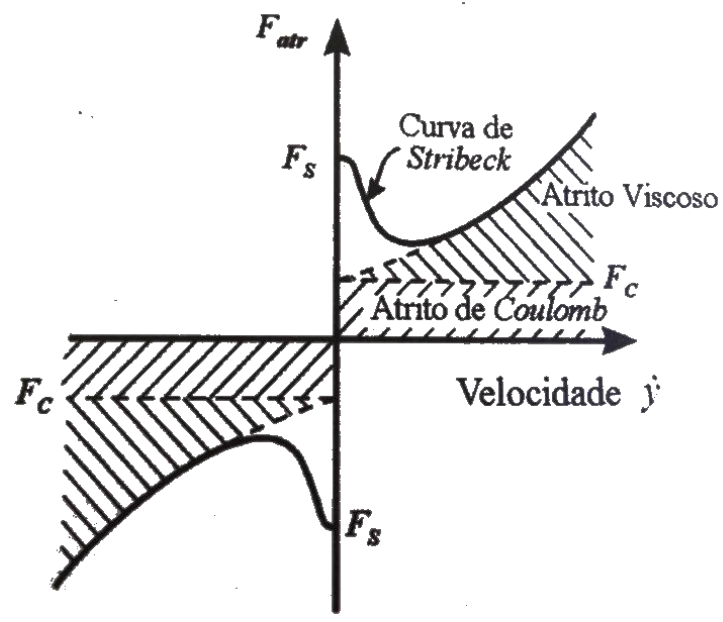

Figura 1 - Combinação das Características do Atrito em Regime Permanente Fonte: Valdiero, 2012.

Um grande avanço na modelagem do atrito foi proposto por [1]. O modelo dinâmico denominado LuGre, é baseado nas microdeformações que ocorrem entre as superfícies de contato. Quando uma força tangencial é aplicada, as cerdas elásticas se deflexionarão como molas. Se estas deflexões forem suficientemente grandes, as cerdas irão deslizar (stick-slip). A velocidade é que determina a deflexão média das cerdas nos movimentos em regime permanente, a qual é modelada pelo atrito de Stribeck [6]. O atrito dinâmico é dado pela equação (1).

$$
F_{\text {atr }}=\sigma_{0} z+\sigma_{1} \dot{z}+\sigma_{2} \dot{y}
$$

onde $F_{a t r}$ é a força de atrito dinâmico do modelo LuGre [1]. Os parâmetros dinâmicos $\sigma_{0}$ e $\sigma_{1}$ são o coeficiente de rigidez das deformações microscópicas entre as superfícies em contato durante o regime de predeslizamento e o coeficiente de amortecimento associado à taxa de variação de $z$ (ou seja, $\dot{z}$ ), respectivamente. O parâmetro estático $\sigma_{2}$ é o coeficiente de amortecimento viscoso $(B)$. A dinâmica do estado interno do atrito é denominado por $z$, o qual descreve a deflexão média das superfícies em contato durante a fase de atrito estático, ou, em outras palavras, a deformação do movimento de predeslizamento. Por fim, o parâmetro $\dot{y}$ representa a velocidade relativa entre as superfícies. A equação (1) pode ser reescrita através da equação (2) que descreve o mapa estático do atrito contemplando as características descritas

$$
F_{a t r}=\left(F_{c}+\left(F_{s}-F_{c}\right) e^{-\left(\frac{\dot{y}}{\dot{y}_{s}}\right)^{2}}\right) \operatorname{sgn}(\dot{y})+\sigma_{2} \dot{y}
$$

onde $F_{c}$ é a força de atrito de Coulomb, $F_{S}$ é a força de atrito estático e $\dot{y}_{s}$ é a velocidade de Stribeck.

Este modelo pode ser incorporado no modelo dinâmico da castanha do fuso de esferas, o qual é descrito através da equação (3) e que foi obtido através do método de Newton-Euler a partir do equilíbrio dinâmico no diagrama de corpo livre [7].

$$
M_{e q} \ddot{y}+F_{a t r_{e q}}=\frac{2 \pi}{p} T_{m}
$$


A equação (3) indica que a massa deslocada sobre o eixo fuso $\left(M_{e q}\right)$ depende da aceleração $(\ddot{y})$ adicionada à força de atrito $\left(F_{a t r_{e q}}\right.$ descrita na equação (2)) resulta na força de reação que depende do passo $(p)$ e do torque motor aplicado $\left(T_{m}\right)$.

\section{Método}

Esta pesquisa é descritiva e exploratória classificando-se como bibliográfica e experimental. Para as simulações foi construído o protótipo de uma junta do robô Gantry com fuso de esferas sendo conectados sensores de deslocamento linear e angular. O robô será conectado a uma placa alemã dSPACE DS 1104, que integrada a um microcomputador, faz a aquisição dos sinais dos sensores e possui interface com o Simulink/MatLab ${ }^{\circledR}$ através do ControlDesk.

Os testes consistiam da regulagem de um potenciômetro em uma determinada marcação, a qual representava uma determinada velocidade de execução do movimento. Em cada marcação era executado o deslocamento positivo e negativo, variando a em cada teste a velocidade, conforme a viabilidade da instrumentação, da mais baixa a mais alta. Em cada teste são coletados os dados obtido através do ControlDesk e da instrumentação (wattímetro, voltímetro e amperímetro).

Em cada experimento foi gerado o gráfico de seu deslocamento e posteriormente, no trecho onde a velocidade é constante, ajustou-se uma reta. De posse das informações da velocidade (coeficiente angular de cada reta ajustada), estimou-se o torque motor. Para isso, utilizou-se a informação do fabricante sobre o rendimento do motorredutor CA para estimar o torque. Como em nenhuma das velocidades desempenhadas, o motor ultrapassou $50 \%$ de sua potência máxima utilizou-se um rendimento $(\eta)$ constante dado pelo produto entre o rendimento do motor (74\%) e do redutor (78\%), para o cálculo da potência mecânica.

De posse dos dados experimentais, o modelo matemático da equação (3) foi implementado em diagrama de blocos com o mapa estático de atrito e simulado computacionalmente para verificar a acurácia do modelo em detrimento aos testes experimentais realizados. Nas simulações não foi considerada a dinâmica elétrica do sistema. As simulações serão realizadas através da ferramenta de prototipagem matemática Simulink/MatLab ${ }^{\circledR}$.

\section{Resultados}

Para a identificação da não linearidade do atrito, foram realizados testes experimentais no protótipo do robô Gantry. Para a velocidade em regime permanente de cada simulação ajustou-se uma reta a qual se faz necessário para melhorar o ajuste dos parâmetros estáticos de atrito, além de ser possível desconsiderar a inércia dada a nulidade da aceleração. A força de atrito pode ser obtida pela relação entre o passo do fuso e o torque do motor, que por sua vez foi obtido para cada simulação a partir da relação entre o rendimento, potência mecânica e velocidade em regime permanente.

Os resultados dos testes em regime permanente são representados na forma de pontos no gráfico da Figura 2. 


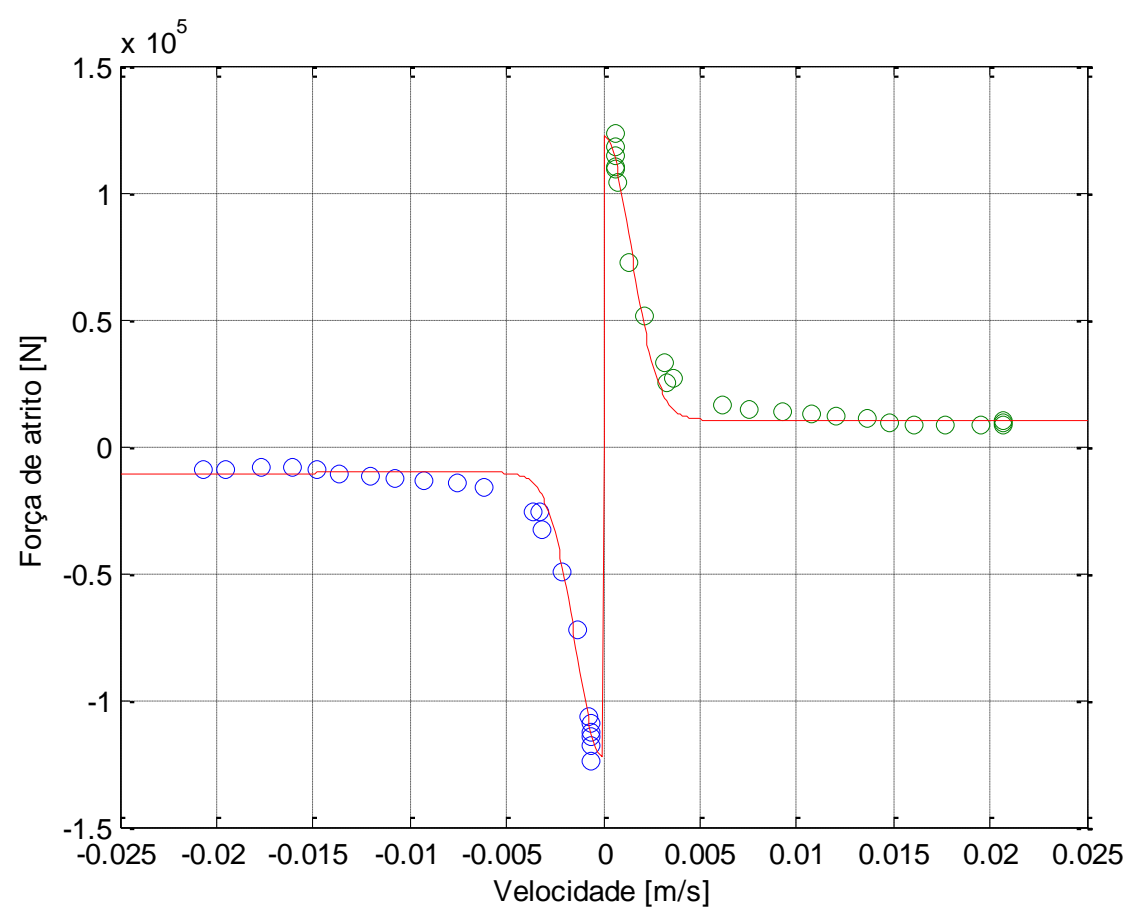

Figura 2 - Mapa Estático do Atrito Relacionando no Eixo $y$ a Força de Atrito $\left(F_{a t r}\right)$ e no Eixo $x$ a Velocidade $\left(\dot{y}_{s}\right)$ em Regime Permanente Fonte: próprio autor.

O ajuste de uma curva ao mapa estático, conforme apresentado na Figura 2, relacionando a velocidade em regime permanente e a força de atrito. Levando em consideração a equação (2) do atrito (mapa estático de atrito) a qual captura as características do atrito estático, atrito de Coulomb, atrito de arraste e o atrito de Stribeck. Para o ajuste utilizou-se a função nlinfit do MatLab e o ajuste por simulações computacionais, de modo a realizar o melhor ajuste dos parâmetros, descritos na Tabela 1.

Tabela 1 - Parâmetros para o Cálculo das Características do Atrito

\begin{tabular}{|c|l|c|c|}
\hline Parâmetro & \multicolumn{1}{|c|}{ Descrição } & Valor & Unidade \\
\hline$F_{c}$ & Coeficiente de atrito de Coulomb & 10300 & $\mathrm{~N}$ \\
\hline$F_{S}$ & Coeficiente de atrito estático & 12300 & $\mathrm{~N}$ \\
\hline$\dot{y}_{s}$ & Velocidade de Stribeck & 0,002 & $\mathrm{~m} / \mathrm{s}$ \\
\hline$\sigma_{2 p}$ & $\begin{array}{l}\text { Coeficiente de amortecimento viscoso (B) } \\
\text { positivo }\end{array}$ & $3,2189 \cdot 10^{3}$ & $\mathrm{Ns}^{2} / \mathrm{m}^{2}$ \\
\hline$\sigma_{2 n}$ & $\begin{array}{l}\text { Coeficiente de amortecimento viscoso (B) } \\
\text { negativo }\end{array}$ & $1,5277 \cdot 10^{4}$ & $\mathrm{Ns^{2 }} / \mathrm{m}^{2}$ \\
\hline$M$ & Massa deslocada & 11,250 & $\mathrm{~kg}$ \\
\hline$J_{m}$ & Momento de inércia do eixo motor & $1.2298 \cdot 10^{-5}$ & $\mathrm{kgm}$ \\
\hline$M_{e q}$ & Massa equivalente & 23,0530 & $\mathrm{~kg}$ \\
\hline
\end{tabular}

Fonte: próprio autor. 
Considerando os parâmetros descritos na Tabela 1Tabela 1 (para o coeficiente de amortecimento viscoso negativo $\left(\sigma_{2 n}\right)$ ) para se fazer a simulação utilizando o Simulink/MatLab, a partir da escrita do diagrama de blocos referente as equações (2) e (3), pode-se validar o modelo para um dos experimentos conforme a Figura 3

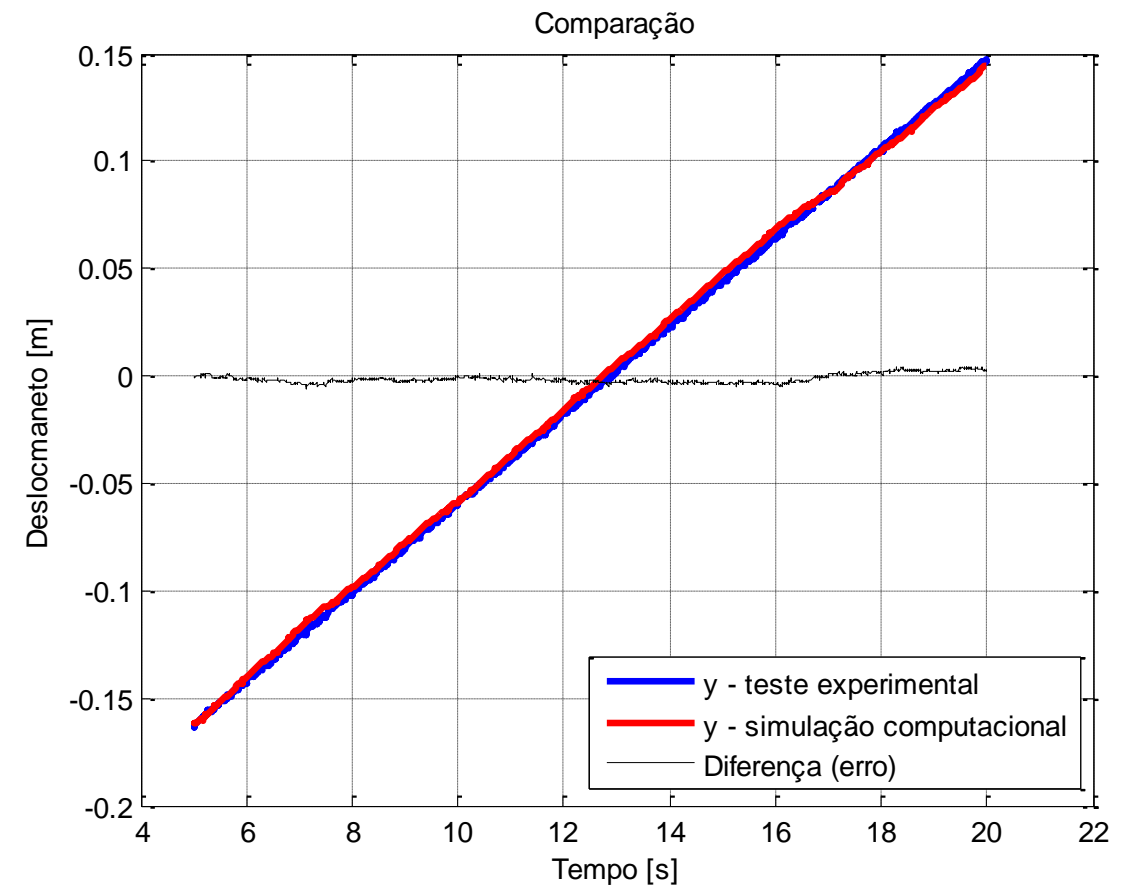

Figura 3 - Validação do Modelo Dinâmico Com a Inclusão das Características do Mapa Estático de Atrito

Fonte: próprio autor.

É relevante notar que o erro obtido é próximo de zero ao longo de todo o experimento, o que indica a acurácia do modelo adotado com a inclusão do mapa estático de atrito.

\section{Discussões}

Apresentou-se neste trabalho a identificação a não linearidade de atrito e a inclusão desta na modelagem dinâmica do protótipo de uma junta de robô do tipo Gantry acionado por inversor de frequência e motorredutor de corrente alternada. Foi construído um protótipo e identificado experimentalmente às características do atrito (as quais estão apresentadas no mapa estático de atrito) e feita à inclusão destas características no modelo dinâmico do robô Gantry o qual é validado experimentalmente. O mapa estático de atrito indica a característica do atrito de Coulomb é mais relevante em detrimento ao atrito viscoso convencionado pela literatura. Na comparação apresentada na Figura 3, entre o teste experimental e a simulação computacional, percebe-se que o modelo descreve com acurácia a dinâmica da junta robótica o que pode contribuir no desenvolvimento de estratégias de controle mais precisas e por consequência, na 
melhoria da qualidade dos processos além de contribuir para a robotização de baixo custo nas mais diversas aplicações, como a marcenaria e a usinagem.

\section{Agradecimentos}

Aos autores são agradecidos a CAPES pela bolsa de mestrado e à Unijuí pela estrutura laboratorial disponível no NIMASS do Câmpus Panambi, implantado com apoio financeiro do FINEP, SEBRAE, CNPq, FAPERGS e MCT.

\section{Referências}

[1] C. Canudas de Wit, H. Olsson, K. J. Åström and P. Lischinsky. A New Model for Control Systems with Friction, IEEE Transactions on Automatic Control, vol. 40, n. 3, 419425, (1995), DOI: 10.1109/9.376053.

[2] L. R. Douat, I. Queinnec, G. Garcia and M. Michelin. Identification and Vibration Attenuation for the Parallel Robot Par2, IEEE Transactions on Control Systems Technology, vol. 22, n. 1, 190-200, (2014), DOI: 10.1109/TCST.2013.2249515.

[3] M. Hanifzadegan and R. Nagamune. Tracking and Structural Vibrtion Control of Flexible Ball-Screw Drives with Dynamic Variations, IEEE/ASME Transactions on Mechatronics, vol. 20, 133-142, (2015), DOI: 10.1109/TMECH.2014.2298241.

[4] S. PAATZ. Anatomy of a Robot. Engineering \& Technology, vol. 3, 42-44. (2008), DOI 10.1049/et:20080113.

[5] W. Po-Nagen. Real-time Inter-processing for PC Based Robot Controllers in Telerobotic and Gantry Robotic Controller System, International Conference on Robotics and Biomimetics, vol. 1, 1585-1589, (2009), DOI: 10.1109/ROBIO.2009.4913237.

[6] A. S. Sanca. Controle com Compensação de Atrito para Estruturas de Base Móveis de Robôs Manipuladores, Dissertação de Mestrado em Engenharia Elétrica, UFBA, (2006).

[7] L. Sciavicco, B. Siciliano. Modeling and Control of Robot Manipulators, McGraw-Hill, (1996).

[8] N. Shimada, T. Yoshioka, K. Ohishi, M. Toshimasa And Y. Yokokura. Reliable Forcesensorless Contact Detection Method for Ball Screw Drive Cartesian Robot, IEEE International Symposium on Industrial Electronics (ISIE), vol. 1, 1-6, (2013), DOI: 10.1109/ISIE.2013.6563694.

[9] A. C. Valdiero. Modelagem Matemática de Robôs Hidráulicos, Unijuí, (2012). 